\title{
Biomimetic Lateral-Line System for Underwater Vehicles
}

\author{
Jan-Moritz P. Franosch*, Stefan Sosnowski ${ }^{\dagger}$, Nader Kuhenuri Chami ${ }^{\dagger}$, \\ Kolja Kühnlenz ${ }^{\dagger}$, Sandra Hirche ${ }^{\dagger}$, J. Leo van Hemmen* \\ * Physik Department T35, Technische Universität München, 85747 Garching bei München, Germany \\ Email: Jan-Moritz.Franosch@ph.tum.de \& 1vh@tum.de \\ $\dagger$ Institute of Automatic Control Engineering, Technische Universität München, 80290 München, Germany \\ Email: sosnowski@tum.de
}

\begin{abstract}
Fish detect underwater objects by measuring even tiny water velocity changes on their skin through their lateral-line system. Several approaches try to mimic the lateral-line system by hot-wire or electro-mechanical sensor arrays but none has achieved yet an underwater vehicle using a biomimetic lateralline system for object avoidance, not even in simulation. In this paper we describe construction and properties of low-cost sensors applicable to the task, viz., sensors where a constant current heats thermistors in water flow. With increasing stream velocity, thermal dissipation and thus voltage increases. A glassbead and a bow protect the thermistors. The small diameter of $0.36 \mathrm{~mm}$ results in a small time constant and thus allows fast measurements. Sensors are about $1 \mathrm{~cm}$ long and consume less than $0.2 \mathrm{~W}$ so that our small-sized underwater vehicle prototype "Snookie" can carry a whole array of them. We describe the magnitude of velocity changes depending on the distance to an obstacle and show experimentally that a single sensor can already detect underwater objects - so much the better a whole ensemble of them, as in fish.
\end{abstract}

\section{INTRODUCTION}

Fish often use their lateral-line system as their only means of navigation, especially under poor visual conditions. An explicit example is the blind Mexican cave fish that, though without vision, is able to navigate around objects. It appears to "perceive" the objects as it passes by [1]-[3]. It does so by detecting tiny water velocity changes on its skin that occur when the fish passes nearby obstacles. For stationary objects, the lateral-line system only works in a very close-up range to the objects to be detected, say, in a distance of about one fish length [4]. Moving objects, on the other hand, often generate wakes and mark their trajectories through a vortex street [5] a strategy that could be pursued by a technical system as well.

Fields of possible applications for an underwater vehicle equipped with an artificial lateral-line system could be turbid water with very poor vision as in canalization, pits filled up with water, swamped buildings, and deep sea. Even under good visual conditions, the lateral-line system may provide useful information to avoid collisions. Object detection by a lateral-line system requires only passive sensors, which has the advantage that sensors do not interfere with each other as, e.g., in a swarm of vehicles.

Underwater vehicle Snookie has been built to mimic fish navigation; cf. Fig. 1. It shall avoid objects by measuring water velocity at different positions on its nose. Artificial
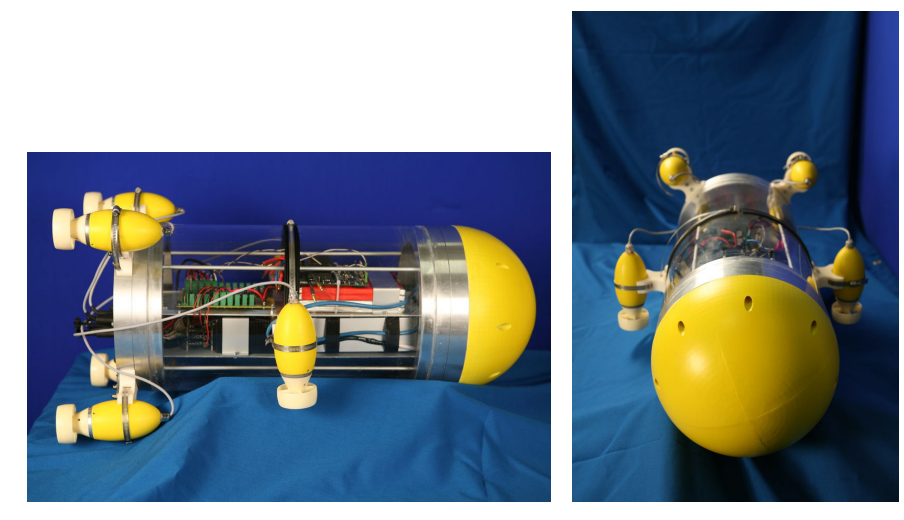

Fig. 1. Prototype of underwater vehicle "Snookie". Velocity sensors will be mounted onto the yellow nose.

lateral-line systems are under research [6]-[8], but have not yet been used as a robotic sensory system. Instead of using mechanoreceptors, Snookie will use hot-thermistor anemometry to measure water velocities. Hot-wire anemometry to measure fluid velocities is well established [9]-[14], also by means of microelectromechanical devices [15], [16]. Hotthermistor anemometry has long been used to measure air and water velocities [17]-[22]. The novel sensor concept has been tested in past experiments with a sphere dragged through the water in the vicinity of a wall [23], but neither sensors usable in an autonomous vehicle have been developed nor has it been shown that object detection is possible when passing an obstacle.

The contribution of this paper is to describe the construction and properties of low-cost hot-thermistor sensors suitable for a biomimetic lateral-line system that can be used in an underwater vehicle. Applicable water-velocity sensors have to resolve tiny changes of velocity fast enough, be robust so that they will not be damaged during accidental collisions or by touch, and underwater function has to be stable. Sensors should consume little power and be small in size so that is is possible to mount ten to twenty of them onto the hull of a small vehicle. In the following we show that the sensors described here fulfill all the above requirements.

The remainder of this paper is organized as follows. In section II we estimate expected velocity changes on Snookie's 
hull when it approaches a wall. In section III we describe sensor construction. We show through simulations that sensor packaging hardly influences sensitivity. In section IV we describe measurement results on the physical properties of the sensors. We give an account of an experiment where a sensor clearly responds to increased water velocity caused by the presence of the object.

\section{Estimating Expected Water Velocities}

For the sake of simplicity and to be able to derive analytic results, we approximate the underwater vehicle Snookie by a sphere. A sphere of radius $a$ moving with velocity $\mathbf{V}$ in a nonviscous fluid generates a so-called dipolar velocity field. If the sphere is at the origin of the coordinate system, the water velocity $\mathbf{v}$ at position $\mathbf{r}$ generated by the sphere is [24]

$$
\mathbf{v}(\mathbf{r}, \mathbf{V})=\frac{a^{3}}{2|\mathbf{r}|^{5}}\left[3(\mathbf{V} \cdot \mathbf{r}) \mathbf{r}-|\mathbf{r}|^{2} \mathbf{V}\right]
$$

Now let Snookie approach a wall. An infinite wall introduces another boundary condition, viz., that the fluid at the wall can only move parallel to it. As described elsewhere [23], we can fulfill the additional boundary condition by introducing a "mirror" sphere as indicated in Fig. 2. We get the mirror sphere by mirroring the original's sphere position and velocity by using the wall as a mirror. Because of mirror symmetry, the velocity field generated by both spheres at the wall is then parallel to the wall, conforming to the Euler boundary conditions that fluid can only move tangentially to a boundary.

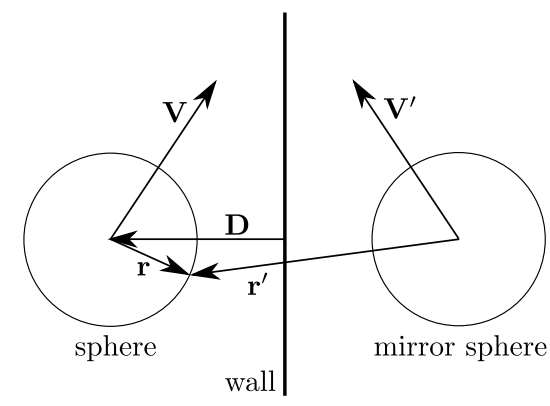

Fig. 2. A sphere is moving with given velocity $\mathbf{V}$ towards a wall. The mirror sphere moving with velocity $\mathbf{V}^{\prime}$ serves to fulfill the boundary condition at the wall. A velocity sensor sits at position $\mathbf{r}$ on the sphere's surface.

The velocities caused by the moving sphere and by its mirror sphere add up linearly so that the overall water velocity at position $\mathbf{r}$, in the reference frame of the fluid, is

$$
\mathbf{v}=\mathbf{v}(\mathbf{r}, \mathbf{V})+\mathbf{v}\left(2 \mathbf{D}+\mathbf{r}, \mathbf{V}^{\prime}\right) .
$$

As $\mathbf{v}$ is not necessarily tangential to the surface, this velocity has still to be projected onto the surface to get an estimate of the velocity at the surface. As the thermistor sensors sit under a protective bow, each sensor has a preferred direction $\mathbf{s}$, with $|\mathbf{s}|=1$ and $\mathbf{s}$ tangential to the surface of the sphere. In addition, the sphere itself is moving with velocity $\mathbf{V}$. Thus the water velocity $v_{s}$ measured by a sensor on the surface of the vehicle is

$$
v_{s}=(\mathbf{v}-\mathbf{V}) \cdot \mathbf{s}
$$

Figure 3 shows the change of water velocity on the surface of a spherical vehicle approaching a wall.
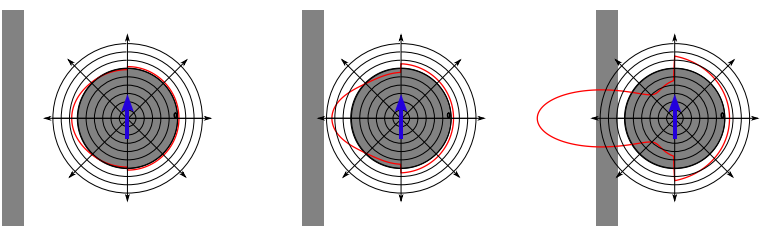

Fig. 3. Fluid velocity differences (red, magnified) between a flow-velocity field around a freely moving sphere and disturbed by a wall. The magnitude of velocity change is about $1 \%$ at one radius distance (left).

The above approach is an approximation insofar as the boundary condition at the surface of the first sphere is disturbed by the presence of the second sphere and thus boundary conditions on the spheres are only satisfied approximately.

\section{ARTIFICIAL LATERAL-Line SyStem USING HOT-THERMISTOR ANEMOMETRY}

To detect and avoid underwater objects, Snookie shall be equipped with an array of sensors to measure water velocity at several places on its nose. The authors have constructed hot-thermistor based water-velocity sensors described in the following.

\section{A. Hot-Thermistor Anemometry}

If a thermistor has temperature $T$, the surrounding water has temperature $T_{\infty}$ and moves with velocity $v$, then energy dissipation $P$ at the thermistor can be described by [10], [11], [14]

$$
P=\left[A+B v^{n}\right]\left(T-T_{\infty}\right)
$$

where $A, B$ and $n$ are constants depending on size and shape of the thermistor and the surrounding medium. Thus, the faster the water velocity, the higher the energy dissipation and therefore energy dissipation can be used to measure water velocity. With the specific heat capacity $c_{p}$, heat conductivity $k$, kinematic viscosity $\nu$, we obtain for a sphere of diameter $d$ [20], [22],

$$
\begin{aligned}
P \approx & {\left[2+0.55\left(\frac{\nu c_{p} \rho}{k}\right)^{0.33}\left(\frac{v d}{\nu}\right)^{0.5}\right] } \\
& 4 \pi\left(\frac{d}{2}\right)^{2} \frac{k}{d}\left(T-T_{\infty}\right) .
\end{aligned}
$$

The glass-bead thermistors used here are nearly spherical and have diameters of $0.36 \mathrm{~mm}$. For a sphere with diameter $d=$ $0.36 \mathrm{~mm}$ in water, $n \approx 0.5$, and, using the constants from Table $\mathrm{I}, A \approx 1.35 \mathrm{~mW} / \mathrm{K}$ and $B \approx 13.5 \mathrm{~mW} /(\mathrm{K} \sqrt{\mathrm{m} / \mathrm{s}})$.

\section{B. Thermistor Coating and Sensor Assembly}

Thermistors (Honeywell 111 Series, hermetically sealed glass-bead, diameter $0.36 \mathrm{~mm}, \beta_{\theta}=-3090 \mathrm{~K}, R\left(20^{\circ} \mathrm{C}\right) \approx$ $2 \mathrm{k} \Omega$ ) were soldered onto small boards $1 \times 1 \mathrm{~cm}$ in size; cf. Fig. 4.

All connection wires were coated with PCB lacquer (Leiterplattenlack ISO-RC 4000, ISO-Elektra Elektrochemische 
TABLE I

HYDRODYNAMIC CONSTANTS OF WATER AND AIR AT $20^{\circ} \mathrm{C}$.

\begin{tabular}{|c|c|c|c|}
\hline & air & water & unit \\
\hline \hline specific heat capacity $c_{p}$ & 1006 & 4182 & $\mathrm{~J} /(\mathrm{kg} \mathrm{K})$ \\
\hline density $\varrho$ & 1.204 & 998 & $\mathrm{~kg} / \mathrm{m}^{3}$ \\
\hline heat conductivity $k$ & 0.0256 & 0.604 & $\mathrm{~W} / \mathrm{Km}$ \\
\hline kinematic viscosity $\nu$ & $1.511 \cdot 10^{-5}$ & $0.979 \cdot 10^{-6}$ & $\mathrm{~m}^{2} / \mathrm{s}$ \\
\hline
\end{tabular}

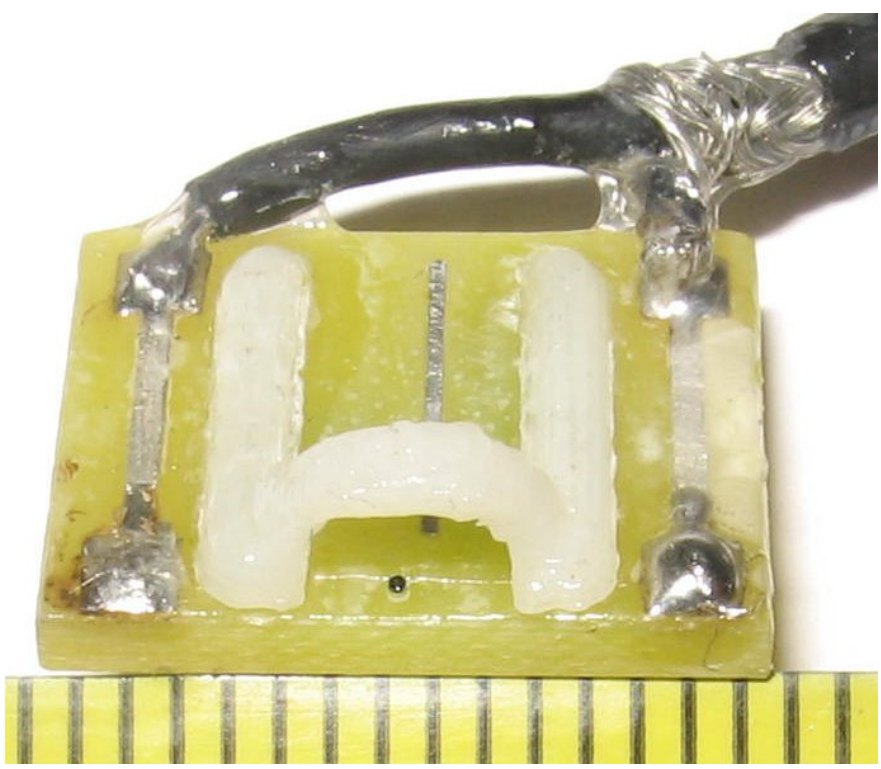

Fig. 4. The black thermistor (diameter $0.36 \mathrm{~mm}$ ) sits under a protective bow. The yellow scale below indicates millimeters.

Fabrik $\mathrm{GmbH}$ ). The lacquer was applied with a thin paint brush carefully covering the connection wires but not the thermistor itself. Electrical isolation was checked with a conductive dilution of salt in water by measuring the resistance between lacquer-covered connections and a reference electrode that was 1-3 $\mathrm{M} \Omega$ compared to about $30 \mathrm{k} \Omega$ for a blank connection. It is very important that the sensor is electrically isolated from water to prevent electrolysis. Electrolysis starts at about $1.5 \mathrm{~V}$ [25] and causes bubbles that may well influence the effective thermal conductivity of the probe.

\section{Thermistor Packaging}

To protect the very fragile thermistors mechanically, especially the thin connection wires, the authors have covered the thermistors with plastic bows printed by a 3D printer (dimension elite); cf. Fig. 4. We have also put the thermistors into canals of about $1 \mathrm{~mm}$ diameter to protect the thermistors even better; cf. Fig. 5. The packaging has the additional advantage that, with only two additional holes and a closed canal, we can easily implement what in biology corresponds to canal organs. An open canal implements a so-called surface lateral-line organ that reacts to velocity whereas a canal organ reacts to pressure difference and hence to acceleration [26].

Simulations (Comsol software package, Navier-Stokes solver) show that packaging the thermistor in a canal hardly
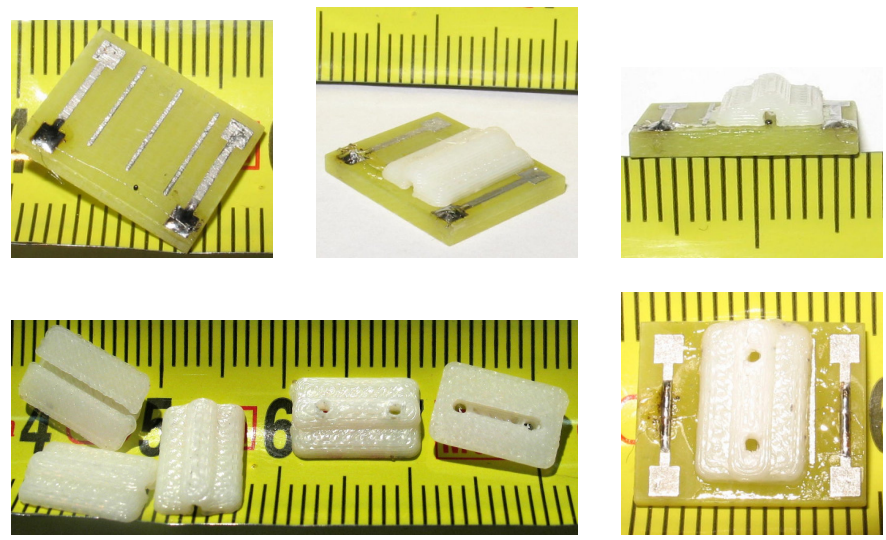

Fig. 5. Thermistors (diameter $0.36 \mathrm{~mm}$ ) mounted on a board, canals to cover the thermistor and a thermistor covered with a plastic canal.

influences heat flux from the thermistor to the water at velocities faster than about $1 \mathrm{~cm} / \mathrm{s}$; cf. Fig. 6 . For these velocities, the fluid boundary layer, i.e., the area where fluid velocity is influenced by the presence of the boundary, is thinner than the diameter of the canal. The presence of the canal therefore does not reduce stream velocity that much. The temperature boundary layer is even thinner than the fluid boundary layer. Thus the presence of the thermally isolating canal does not significantly reduce heat flux from the thermistor to the water.

\section{Color coding for temperatures $(\mathrm{K})$ :}

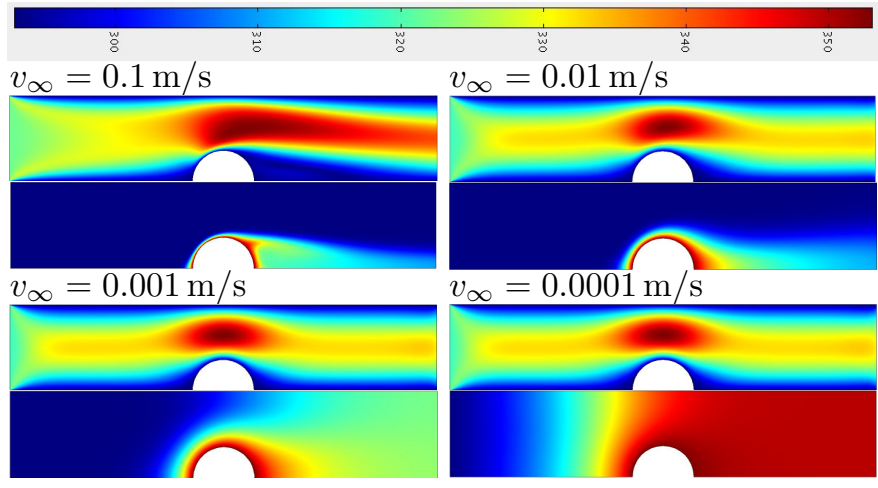

Fig. 6. Simulation of velocity (upper) and temperature (lower layer) distributions for the indicated velocities. The thermistor is in the middle of a canal. For simplicity and computational feasibility, the simulation has been done in $2 \mathrm{D}$ in such a way that the thermistor is a half cylinder with diameter $0.36 \mathrm{~mm}$ in the simulated geometry. The bulk water temperature is $T_{\infty}=293 \mathrm{~K}$, the thermistor temperature $T=353 \mathrm{~K}$, thus over-temperature is $T-T_{\infty}=60 \mathrm{~K}$. Boundary conditions are constant velocity $v_{\infty}$ at the left-hand side of the canal and thermally isolating surfaces.

The proposed packagings having almost no influence on functionality, do protect the thermistor from mechanical damage. According to simulations, energy dissipation follows the power law (3) although the thermistors have been put into canals; cf. Fig. 7.

\section{Measurement Results}

\section{A. Impulse Response}

Figure 8 shows the impulse response of the sensor. The sensor was driven by a constant current (power source Toellner 


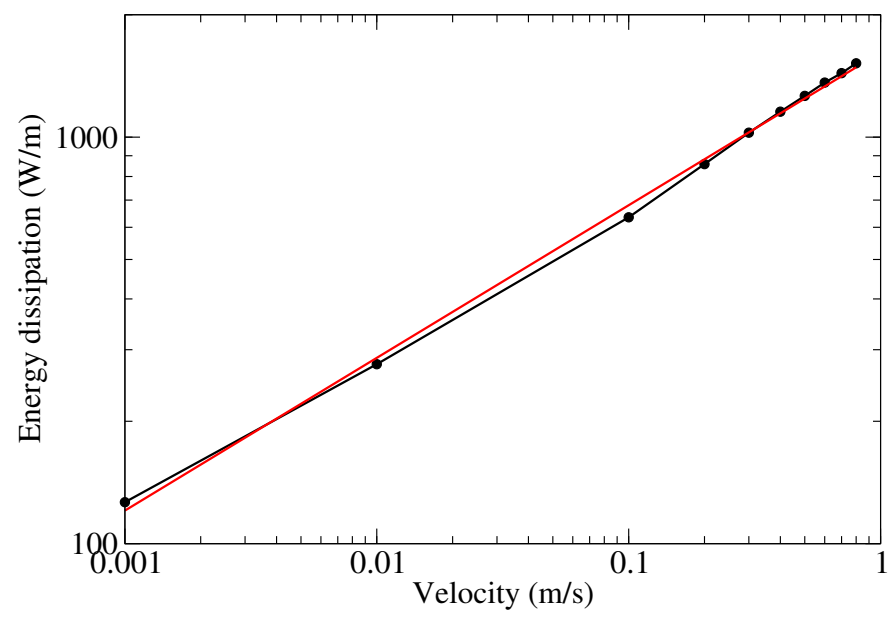

Fig. 7. Black: Simulation results for energy dissipation over velocity in double logarithmic scale. Red: Fit to the data according to the power law $P=B v /(\mathrm{m} / \mathrm{s})^{n}, n=0.38$ and $B=1614 \mathrm{~W} /(\mathrm{Km})$. For thermistor length $0.36 \mathrm{~mm}$, and over-temperature $60 \mathrm{~K}$, this corresponds to $B=9.7 \mathrm{~mW} / K$ in (3) and, regarding the simplifications made and the differences in geometry from a free sphere, fits theory very well.

TOE 8733), kicked by hand much shorter than the typical time constant and the time-dependent voltage was recorded by an oscilloscope (Tektronix TPS 2024). The recording shows that the time constant of sensor response is about $10 \mathrm{~ms}$ as measured from response maximum to half maximum and is thus small enough as to detect water velocity changes in time.

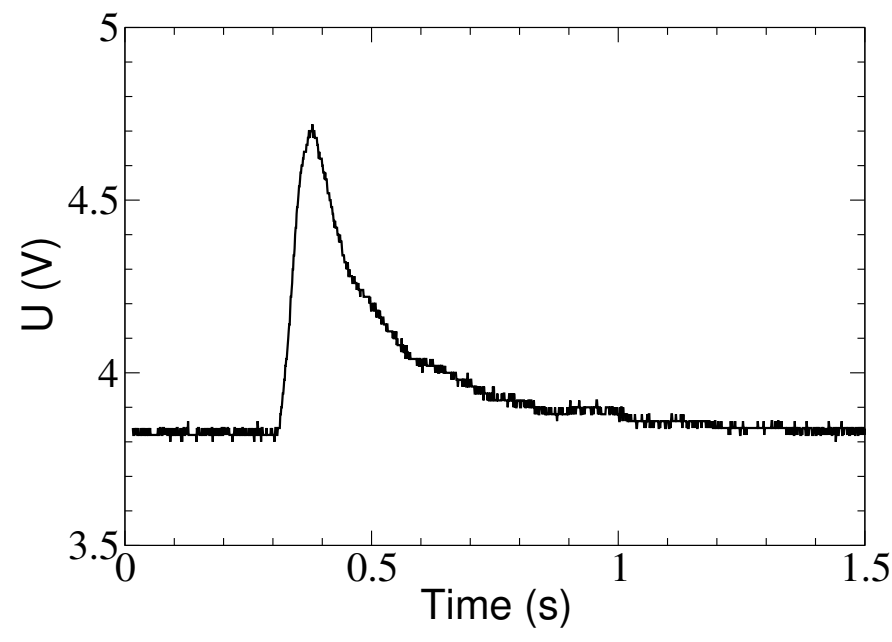

Fig. 8. Voltage impulse response of the thermistor sensor.

\section{B. Energy Dissipation in Dependence upon Over-Temperature}

The authors have measured energy dissipation $P$ in dependence upon the thermistor over-temperature $T-T_{\infty}$ by applying different constant currents (power source Toellner TOE 8733, ampere meter Fluke 45) and recording the voltages across the thermistor through an oscilloscope. According to (3), there should be a linear relation between power $P$ and over-temperature $T-T_{\infty}$ which is indeed the case; cf. Fig. 9.

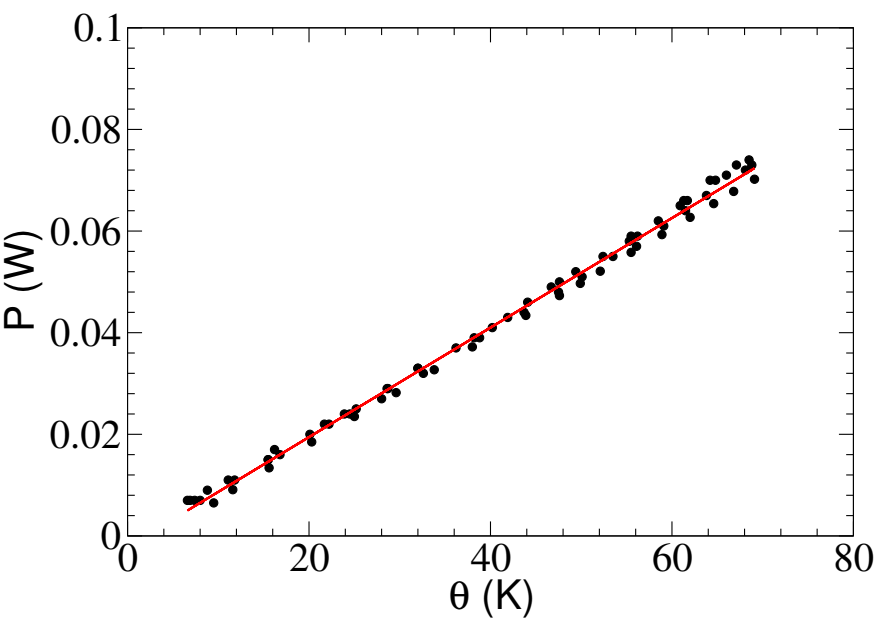

Fig. 9. Black dots: Measurements of energy dissipation $P$ of a thermistor $\left(R_{0}=1523 \Omega\right.$ at $\left.T_{0} \approx 293 \mathrm{~K}\right)$ in water mounted on a PCB board and coated, at different over-temperatures $\theta=T-T_{\infty}$; see (3). Red line: Linear fit to the data. The relation between energy dissipation and over-temperature is perfectly linear $(1.8 \mathrm{~mW} / \mathrm{K})$, as the theoretical relation (3) predicts.

\section{Energy Dissipation in Dependence upon Water Velocity}

To measure energy dissipation $P$ in dependence upon water velocity $v$, the thermistor was driven by a constant current (power source Toellner TOE 8733, ampere meter Fluke 45). A linear axis (Copley Controls Corp, velocity controlled by a Matlab/Simulink program under RTAI realtime Linux) dragged the thermistor sensor through a water basin at constant speed $v$; cf. Fig. 10. The time-dependent voltage has been measured by an oscilloscope for different speeds $v$. Using voltage and current, we have calculated thermal power $P$. Measurement data fit the power law (3) very well; cf. Fig. 11. However, the fitted parameter $B$ in (3) was much smaller than predicted by theory (4) and by simulation; cf. Fig. 7 .

Using the power law (3) and linearizing the temperaturedependent resistance of the thermistor according to

$$
R_{\vartheta}=R_{0} \exp \left[\beta_{\vartheta}\left(1 / T_{0}-1 / T\right)\right]
$$

around the resistance $R_{0}$ at the equilibrium temperature $T_{0}$ for velocity $v=0$, we find a voltage difference

$$
\Delta U \sim B v^{n}
$$

with respect to $v=0$ for small velocities $v$, i.e., a power law with the same coefficient as in (3). Figure 12 shows that measurement data agree with theory.

\section{Object Detection}

To test whether object detection is feasible, the authors have mounted a sensor on the surface of a sphere and dragged it past a cylindrical object; cf. Fig. 13.

Dragging the sensor mounted on a sphere past a cylindrical column results in a clear signal, i.e., an increase in voltage in the vicinity of the object; cf. Fig. 14. The signal contains quite a bit of noise, mainly due to a non-constant velocity of the linear axis, which the authors expect to reduce in future measurements. 


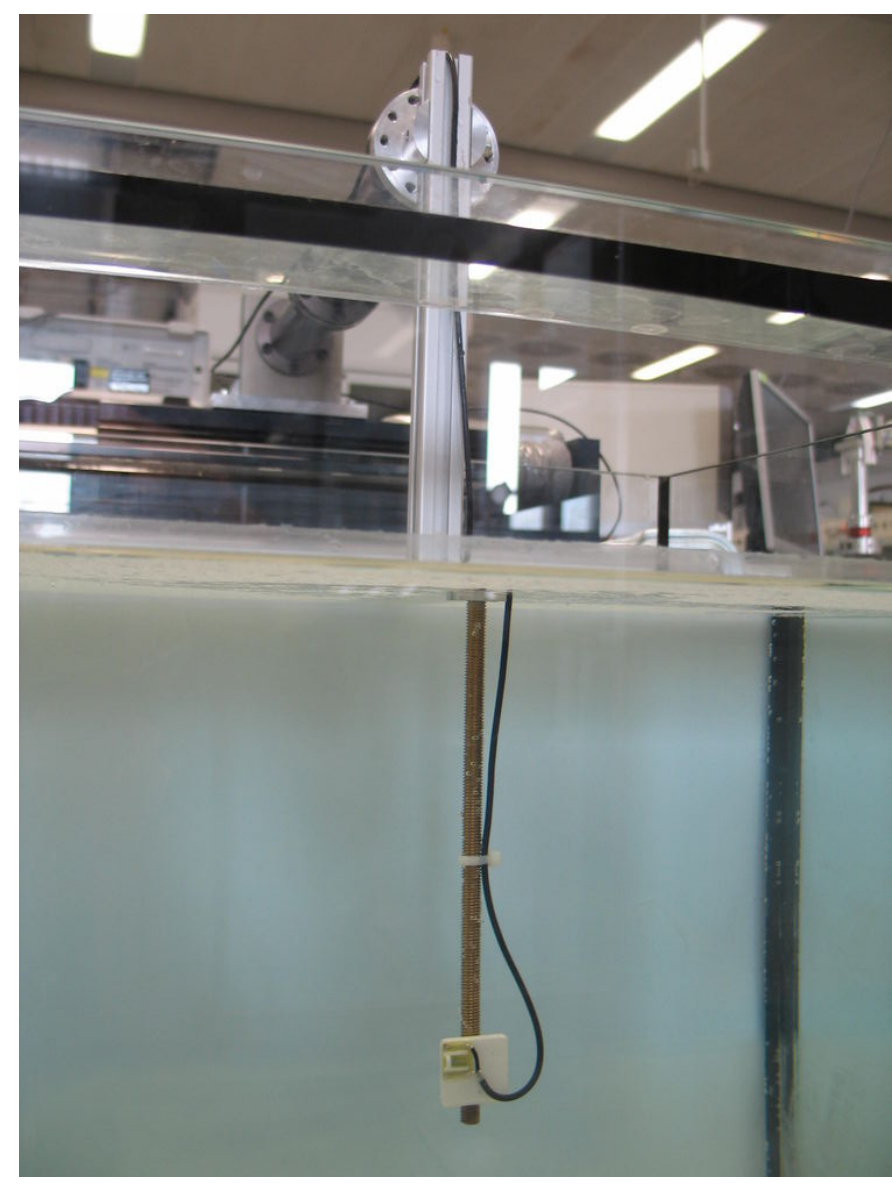

Fig. 10. Measurement apparatus. A linear axis (top) drags the sensor (bottom) mounted on a rod through the water basin.

\section{CONCLUSIONS}

Simulations have shown that packaging the thermistor sensors even into narrow canals does not reduce sensitivity. However, in preliminary experiments performed by the authors the canal-covered thermistors (Fig. 5) did not function properly because the narrow canal quickly filled with gas isolating the thermistor from the water. The authors hope to overcome this problem by covering the canals with hydrophilic lacquer. On the other hand, the bow-protected thermistors of Fig. 4 work well and are ready to use. In small series they can be reproduced quickly and at low cost. That is, 6 euros for material and 15 minutes time for assembly.

The sensors show the physical properties predicted by theory (3). The constant $B$, however, has been found to be much smaller than predicted by both simulations and theory (4). Further measurements have to be done to clarify this point.

Preliminary object detection trials, as in Figs. 13 and 14, show that the sensors work in an object-detection situation. Thus even a single copy of the present water velocity sensors can already detect objects. The authors expect that a biomimetic lateral-line array of sensors can serve as a nearrange collision warning system for underwater vehicles.

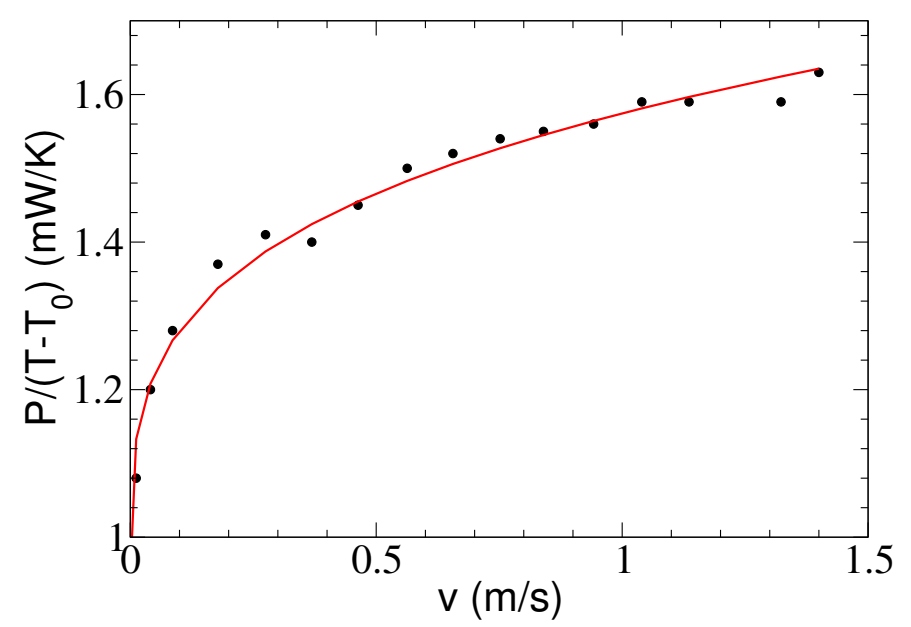

Fig. 11. Black dots: Measurements of the energy dissipation $P /\left(T-T_{\infty}\right)$ per over-temperature of the thermistor sensor from Fig. 12. Red: Fit to the data according to the power law $P /\left(T-T_{\infty}\right)=A+B \cdot[v /(\mathrm{m} / \mathrm{s})]^{n}$ with $n=0.34, A=1.03 \mathrm{~mW} / \mathrm{K}$, and $B=0.74 \mathrm{~mW} / \mathrm{K}$. The data fit the power law (3) rather well, but the parameter $B$ is much smaller than $B \approx 13.5 \mathrm{~mW} /(\mathrm{K} \sqrt{\mathrm{m} / \mathrm{s}})$ predicted by theory (4).

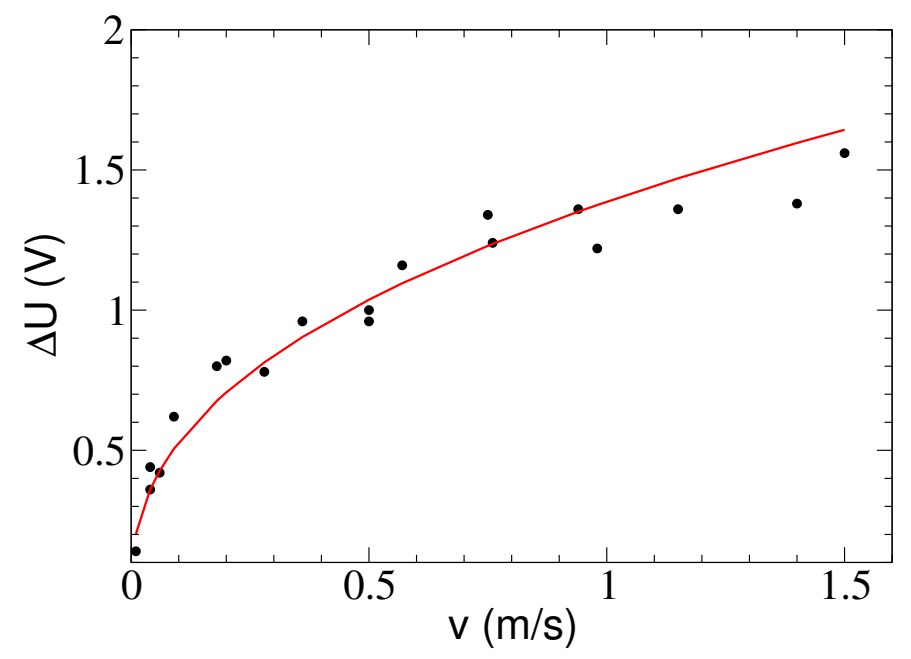

Fig. 12. Black dots: Measurements of the voltage change $\Delta U$ at a thermistor sensor driven by a constant current of $18.1 \mathrm{~mA}$. The voltage across the thermistor was $3.88 \mathrm{~V}$ for water velocity $v=0$. Red: Fit the data according to the power law $\Delta U / \mathrm{V}=B \cdot[v /(\mathrm{m} / \mathrm{s})]^{n}$ with $n=0.42, B=1.39$. The data agree with a power law and thus with theory as in (6).

\section{ACKNOWLEDGMENTS}

The authors thank all students who have contributed to the autonomous underwater vehicle project. This work is supported in part by the DFG excellence initiative research cluster Cognition for Technical Systems - CoTeSys, see also http://www . cotesys.org, and by the Bernstein Center for Computational Neuroscience (BCCN) Munich.

\section{REFERENCES}

[1] T. Teyke, "Collision with and avoidance of obstacles by blind cave fish Anoptichthys jordani (Characidae)," Journal of Comparative Physiology A, vol. 157, pp. 837-843, 1985. 


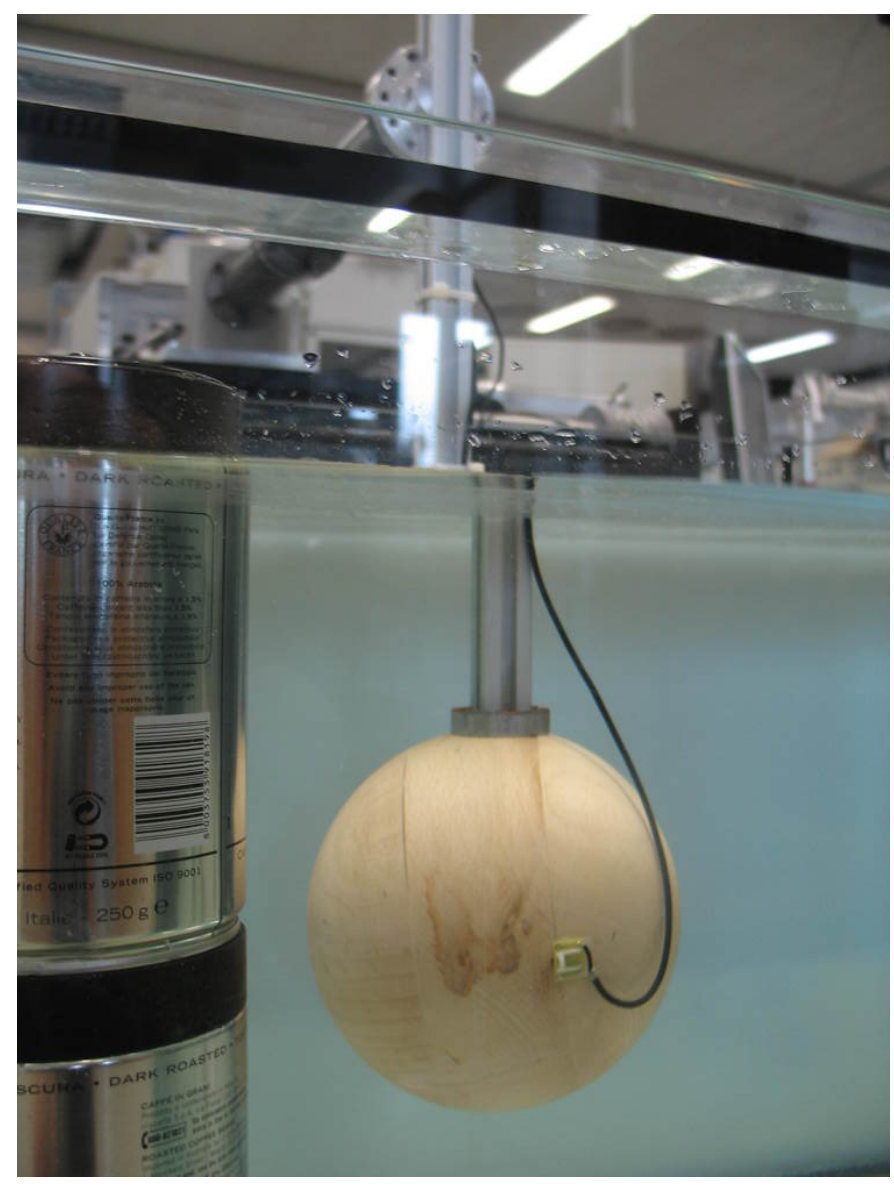

Fig. 13. Setup used for object detection. The linear axis drags a sphere with the sensor past the cylindrical column on the left.

[2] H. Abdel-Latif, E. S. Hassan, and C. von Campenhausen, "Sensory performance of blind Mexican cave fish after destruction of the canal neuromasts," Naturwissenschaften, vol. 77, pp. 237-239, 1990.

[3] E. S. Hassan, H. Abdel-Latif, and R. Biebricher, "Studies on the effects of $\mathrm{Ca}++$ of the blind Mexican cave fish and Co++ on the swimming behavior," J. Comp. Physiol. A, vol. 171, pp. 413-419, 1992.

[4] A. B. Sichert, R. Bamler, and J. L. van Hemmen, "Hydrodynamic object recognition: When multipoles count," Phys. Rev. Lett., vol. 102, p. 058104, 2009.

[5] J.-M. P. Franosch, H. J. A. Hagedorn, J. Goulet, J. Engelmann, and L. J. van Hemmen, "Wake tracking and the detection of vortex rings by the canal lateral line of fish," Phys. Rev. Lett., vol. 103, p. 078102, 2009.

[6] B. Gray, "Artificial arrays could help submarines make like a fish," Science, vol. 313, pp. 1382-1383, 2006.

[7] Y. Yang, J. Chen, J. Engel, S. Pandya, N. Chen, C. Tucker, S. Coombs, D. L. Jones, and C. Liu, "Distant touch hydrodynamic imaging with an artificial lateral line," PNAS, vol. 103, no. 50, pp. 18 891-18 895, 2006.

[8] Y. Yang, N. Nguyen, N. Chen, M. Lockwood, C. Tucker, H. Hu, H. Bleckmann, C. Liu, and D. L. Jones, "Artificial lateral line with biomimetic neuromasts to emulate fish sensing," Bioinspir. Biomim., vol. 5, p. 016001, 2010.

[9] E. L. Piret, W. James, and M. Stacy, "Heat transmission from fine wires to water - low velocity data and correlation," Industrial and Engineering Chemistry, vol. 39, no. 9, pp. 1098-1103, 1947.

[10] H. Strickert, Hitzdraht- und Hitzfilmanemometrie. Berlin: VEB Verlag Technik, 1974.

[11] I. C. Itsweire and K. N. Helland, "A high-performance low-cost constanttemperature hot-wire anemometer," Journal of Physics E - Scientific Instruments, vol. 16, no. 6, pp. 549-553, 1983.

[12] C. G. Lomas, Fundamentals of hot wire anemometry. Cambridge: Cambridge University Press, 1986

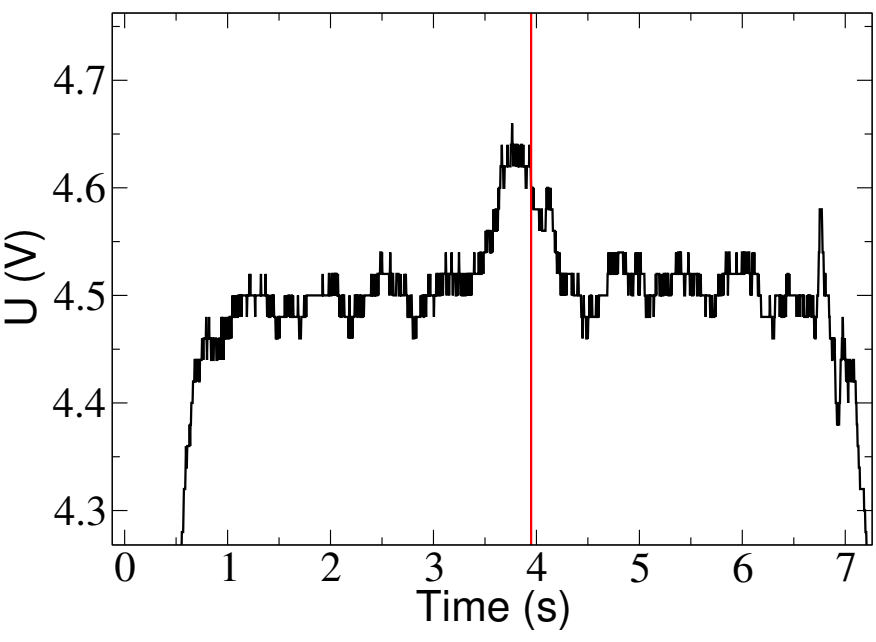

Fig. 14. Voltage response of the sensor in Fig. 13. Cylinder diameter is $9 \mathrm{~cm}$, sphere diameter $15 \mathrm{~cm}$, and velocity $10 \mathrm{~cm} / \mathrm{s}$. The minimal distance between sensor and cylinder is $1.5 \mathrm{~cm}$ and is reached at the time indicated by the red line. The body is clearly detectable by the present apparatus (voltage increases in the vicinity of the red line) although there is still some noise mainly due to the power source (small spikes of about $25 \mathrm{mV}$ coupling into the power source from the linear axis) and a non-constant velocity of the linear axis. The quantization error of the digital oscilloscope is also clearly visible. A bowprotected sensor with $R_{0}=1790 \Omega$ has been used with a constant current of $18 \mathrm{~mA}$.

[13] A. E. Perry, Hot-wire anemometry. Oxford: Clarendon Pr., 1982.

[14] H. H. Bruun, Hot wire anemometry. Oxford: Oxford University Press, 1996.

[15] J. W. van Honschoten, "Modelling and optimisation of the microflown," Ph.D. dissertation, University of Twente, 2004.

[16] J. W. van Honschoten, V. B. Svetovoy, G. M. Krijnen, and M. C. Elwenspoek, "Optimization of a thermal flow sensor for acoustic particle velocity measurements," Journal of Microelectromechanical Systems, vol. 14, pp. 436-443, 2005.

[17] W. Felix, "Strömungsmessung mit Thermistoren," NaunynSchmiedberg's Arch. exp. Path. u. Pharmak., vol. 244, pp. 254-269, 1962.

[18] D. E. Murphy and R. E. Sparks, "A thermistor anemometer for measurement of low fluid velocities," Industrial \& Engineering Chemistry Fundamentals, pp. 642-645, 1968.

[19] F. S. Martino and P. E. McNall, "Thermistor anemometer for measurement of very low air velocities," Review of Sientific Instruments, vol. 42, no. 5, pp. 606-609, 1971.

[20] S. Emsmann and A. Lehmann, "Entwicklung eines Thermistoranemometers zur Messung instationärer Wassergeschwindigkeiten," Fortschrittsberichte der VDI Zeitschriften, vol. 18, no. 8, 1975.

[21] B. Mauconduit and M. Trinite, "New low-speed anemometer with constant temperature thermistor," J. Phys. E: Sci. Instrum., vol. 9, pp. 1091-1096, 1976.

[22] U. Eser, "Thermisches Anemometer mit Kugelsonde zur Bestimmung kleiner Geschwindigkeitsvektoren,” Ph.D. dissertation, Universität Essen, Essen, 1990.

[23] N. Martiny, S. Sosnowski, K. Kühnlenz, S. Hirche, Y. Nie, J.-M. P. Franosch, and J. L. van Hemmen, "Design of a lateral-line sensor for an autonomous underwater vehicle," in 8th IFAC International Conference on Maneuvering and Control of Marine Craft, MCMC 2009, São Paulo, Brazil, 2009, pp. 292-297.

[24] H. Lamb, Hydrodynamics, 6th ed. Cambridge: Cambridge University Press, 1932

[25] G. B. Middlebrook and E. L. Piret, "Hot wire anemometry - solution of some difficulties in measurement of low water velocities," Industrial and Engineering Chemistry, vol. 42, no. 8, 1950.

[26] J. Goulet, J. Engelmann, B. P. Chagnaud, J.-M. P. Franosch, M. D. Suttner, and J. L. van Hemmen, "Object localization through the lateralline system of fish: Theory and experiment," J. Comp. Physiol. A, vol. 194, no. 1, pp. 1-17, 2008. 